\title{
Certain Approach of Object Tracking using Optical Flow Techniques
}

\author{
R. Revathi \\ Research Scholar \\ Karpagam University \\ Coimbatore-21
}

\author{
M. Hemalatha \\ Department of Computer Science \\ Karpagam University \\ Coimbatore-21
}

\begin{abstract}
Now a day's object tracking is more difficult and tricky to surveillance in real time. This proposed work deals with the tracking of moving object in a sequence of frames and it also determines the velocity of the object. In this work algorithms are developed for improving the image quality, segmentation, feature extraction and for identifying the velocity. The algorithms developed are implemented and evaluated using MATLAB. The image quality of the video frame is obtained by applying certain noise removal filters. Next, identifying the moving objects from the portion of the video frame is performed using the background subtraction technique based on frame difference.

The object tracking is performed by optical flow with Bayesian boosting algorithm method on detected object in each frame as a feature extraction method. There are several papers describing the accomplishment of optical flow. Some results are adequate, but in many projects, there are restrictions. In most preceding applications, because the camera is typically static, it is simple to apply optical flow to identify the moving targets in a scene and get their trajectories. When the camera moves a global motion will be added to the local motion, which complicates the issue. In this work we used the combination of boosting algorithm called Bayesian boosting is adopted to improve the performance of Optical flow (OFTBB). The distance traveled by the object is determined using its centroid pixel. It is calculated by using the Euclidean distance formula. Then the velocity of the object is calculated by finding the object moved in distance in a sequence of frames with respect to the video frame rate.
\end{abstract}

\section{Keywords}

Object tracking, Preprocessing, Segmentation, Feature Extraction, Tracking and Detection.

\section{INTRODUCTION}

The process of locating the moving object in sequence of frames is known as Object Tracking. This tracking can be performed by using the feature extraction of object and detecting the objects in sequence of frames.

The process in locating the object which is moving in instance of time is known as video tracking. These are used mainly in the sectors like

1. The interaction between human and computer

2. Security and surveillance

3. Video communication and compression

4. Augmented reality

5. Traffic control

6. Medical imaging [2] and video editing. [3][4].
The goal of video tracking is to combine objects in successive video frames. The connection can be especially difficult when the objects are moving fast virtual to the frame rate. Next is the increase the complication of the problem is tracked when the object changes its direction over the time period. We use video tracking systems to employ a motion model which describes how the image of the target might change for different possible motions of the object.

Video tracking can be a time saving process due to the quantity of data that are contained in video. Adding more to the density is the possibly need to use object recognition techniques for tracking.

\section{MOTIVATION}

The objective of video tracking is to map target objects in consecutive video frames. The mapping can be especially difficult when the objects are moving relatively fast to the frame rate. Another circumstance that raises the obscurity of the problem is when the tracked object changes orientation over time.

\section{APPLICATIONS}

1. Automated Video surveillance: Designed to monitor the movement in an area to identify moving object.

2. Robot Vision: The moving objects obstacles are identified in path to avoid collision in real time object tracking system.

3. Traffic Monitoring: In case of breaks of traffic rules is monitored using cameras is tracked down easily.

4. Animation: Using object tracking algorithm can extend to animation.

\section{RELATED WORKS}

A.Gyaourova, C.Kamath, S.and C.Cheung has studied the block matching technique for object tracking in traffic scenes. A motionless airborne camera is used for video capturing. They have discussed the block matching technique for different resolutions and complexes [5].

Yoav Rosenberg and Michael Werman explain an objecttracking algorithm using moving cameras. The algorithm is based on domain knowledge and motion modeling. Displacement of each point is assigned a discreet probability distribution matrix. Based on the model, image registration step is carried out. The registered image is then compared with the background to track the moving object [6].

A.Turolla, L.Marchesotti and C.S.Regazzoni discuss the camera model consisting of multiple cameras. They use object features gathered from two or more cameras situated at 
different locations. These features are then combined for location estimation in video surveillance systems [7].

One simple feature based object tracking method is explained by Yiwei Wang, John Doherty and Robert Van Dyck.The method first segments the image into foreground are gathered for each object of interest. Then for each consecutive frame the changes in features are calculated for various possible directions of movement. The one that satisfies certain threshold conditions is selected as the position of the object in the next frame [8].

Ci gdem Ero glu Erdem and Bulent San have discussed a feedback -based method for object tracking in presence of occlusion. In this method several performance evaluation measures for tracking are placed in a feedback loop to track non rigid contours in a video sequence [9].

By tracking the object in motion, occlusion Alok K.Watve and Shamik Sural explain and compares the result with various algorithms like Kalman Filtering for foreground extraction and camera modeling for Background Subtraction with multiple cameras in both fixed and moving object. Extracting the feature of tracking object in Block matching calculates the frame difference; in exploiting the domain Knowledge the author calculate the motion parameters with the displacement of scale parameters and displacement vector. In compressed domain object tracking method is calculated using bounding rectangle of an object with different frames by using histogram [10].

P.Subashini,M.Krishnaveni,Vijay Singh discuss the object tracking in Frame Rate Display and Color conversion with background subtraction with different techniques like Estimating median overtime, Computing median overtime, Estimating moving objects. With the comparison of various segmentation algorithms like Sobel Operator, Canny operator and Roberts operator the object is segmented through edge detection and its derivates are calculated. Through region filtering in color segmentation color samples for skin is processed and is computed to mean and covariance over color channels. Object tracking for moving object through motion vector is calculated through optical flow algorithm and Blob analysis for binary feature of an image is calculated. Tracking of object is measures by the position done by tracking in region filtering and the information of the object is created an estimation of new object [11].

Background subtraction and motion estimation for tracking an object.DCT domain background subtraction in Y plane is used to locate candidate objects in subsequent Iframes after a user has marked an object of interest in the given frame.DCT domain histogram matching using $\mathrm{Cb}$ and $\mathrm{Cr}$ planes and motion vectors are used to select the target object from the set of candidate objects [12].

A tracking and detecting algorithm that predicts the object by predictiong the object boundary using block motion vectors followed by updating the contour using occlusions/disclosing detection. Another group of algorithms deals with object tracking using adaptive particle filter [13, 14], kalman filter [15-17].

The efficiency by reducing the number is computations for object tracking in video frames is tracked with the adaptive particle filter, kalman filter and for color frames the image is captured into transform to $\mathrm{YCbCr}$ space with the wavelets coefficient transform for detecting moving objects [18].
A weighted adaptive scalable hierarchical (WASH) tree based video coding algorithm is proposed with low-memory usage. The standard coding uses three separate lists to store and organize tree data structures and their significance, which can grow large at high rate and consume large amounts of memory. In the proposed algorithm, value added adaptive scale down operator discards unnecessary lists and the process length of the sorting phase is shortened to reduce coding time and memory usage. Spatial and temporal scalability can be easily incorporated into the system to meet various types of display parameter requirements and self-adapting rate allocations are automatically achieved. Results show that the proposed method reduces memory usage, run time and improves PSNR [19].

A novel approach for car detection and classification is presented, to a whole new level, by devising a system that takes the video of a vehicle as input, detects and classifies the vehicle based on its make and model. It takes into consideration four prominent features namely Logo of vehicle, its number plate, color and shape. Logo detector and recognizer algorithms are implemented to find the manufacturer of the vehicle. The detection process is based on the Adaboost algorithm, which is a cascade of binary features to rapidly locate and detect logos. The number plate region is localized and extracted using blob extraction method. Then color of the vehicle is retrieved by applying Haar cascade classifier to first localize on the vehicle region and then applying a novel algorithm to find color. Shape of the vehicle is also extracted using blob extraction method. The classification is done by a very efficient algorithm called Support vector machines. Experimental results show that our system is a viable approach and achieves good feature extraction and classification rates across a range of videos with vehicles under different conditions. Edge matching, Divide-and-Conquer search, Gradient matching, Histograms of receptive field responses, Pose clustering, SIFT; SURF etc are some of the approaches applied. All these methods are either Appearance based methods or Feature based methods. They lag in one or the other way when it comes to real time applications. So there has been a need for creating a new system that could combine positive aspects of both the methods and increase the efficiency in tracking objects, when it comes to real life scenario. [20].

Daniel Marcus Jang proposed the Car-Rec search framework is made up of four stages: feature extraction, word quantization, image database search, and structural matching [21]. Car-Rec differs from other car identification systems in that it focuses on efficiently searching a large database of car imagery, using quantized feature descriptors to find a short list of likely matches. This list is then re-ranked with a structural verification algorithm [20].

The merits of this method is that it uses SIFT in place of SURF feature extraction or Lucerne search and the structural verification algorithm may be swapped out for alternative choices. Its modular framework can be applied to general image recognition tasks, particularly with similar image search in large image databases. The demerit of this method is handling imagery with background noise information.

Three methods have been examined: Scale Invariant Feature Transform (SIFT), Principal Component Analysis (PCA), Edge Based Detection. SIFT presented by David Lowe in January, 2004 that has proved to be having a higher level of performance [22]. The features are 3D viewpoint displacement, noise addition and illumination changes. Finding the key points is a 4 step process: Scale Space 
Extreme Detection, Key point Localization, Orientation Assignment, and Descriptor Building.

The SIFT Matching process involves Key point Matching, Clustering, Drawing the Bounding Box. While performing analysis there are two important parameters on which we analyze the ROC curve, DoG Threshold which indicates the minimum contrast a valid key point should have with respect to distance ratio [20].

Optical Character recognition (OCR) is a promising area of research and has wide applications. Character recognition is being an inevitable part in license plate reading, document analysis and OMR sheet reading and most OCR algorithms have been proposed for isolated characters. Geometric or statistical features can be considered for recognition and Eigen feature is one of the statistical features. Abundant literatures are there on Eigen feature based OCR. This paper proposes a Eigen feature regularization and extraction (ERE) algorithm for recognizing characters in number plate of cars. The ERE algorithm reduce the occurrence of false detections. In any character recognition approach recognition errors can be occurred due to presence of noise or due to limited number of training samples. The approach uses K-nearest neighbor classifier to classify the characters. This printed character recognition system shows an excellent accuracy of $98.46 \%$. Experiments are done on different character datasets and are also given [23].

Structure based feature vector is used for machine printed Chinese character recognition in [24]. It uses a minimum distance classifier to classify the test data. Here the recognition accuracy depends on a number of factors such as image resolution, binarization threshold, character normalization etc. and is not robust to noise [23].

Even though several researches are performed on tracking moving objects they suffer from the issue of detecting objects.

\section{OBJECT TRACKING PROPOSED METHODOLOGY}

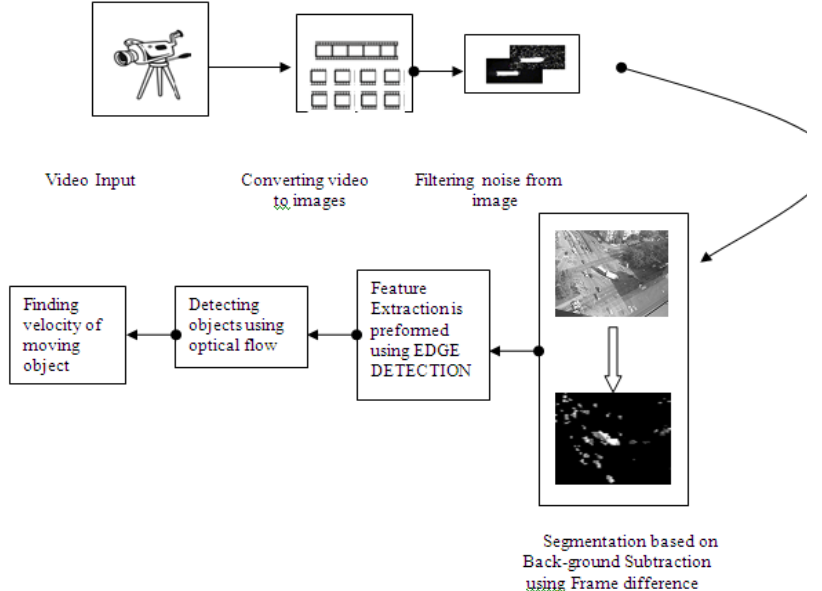

Figure: 1: Architecture of the Proposed Object Tracking Methods

\section{PHASES USED IN OBJECT TRACKING}

\subsection{Pre-processing Phase}

Before performing any video processing operation the quality of the frame is very essential. In this phase improving the quality of frame is taken into the consideration and the video is tested with three types of noises salt and pepper noise, Gaussian noise and periodic noise. These noises have more chances to distract the quality of video frames. The each noise types are denoised using various filtering techniques and the best suited filters are taken into the consideration for different noises.

1. Select three videos which contain three different noises like -Salt and pepper noise/ Gaussian noise /periodic noise.

2. Convert videos to Frames

3. Apply various filters in the noise generated frames

4. Identify the best suited filter using the PSNR and MSE

5. Use the resultant frames for further processing.

\subsection{Segmentation Phase}

To classify the foreground object from the background segmentation operation is performed by background modeling technique, frame differencing. It takes less processing time. The steps are:

1. Read the first input frame and consider it as background frame

2. Convert the background frame to grey scale

3. Set the Threshold value

4. Set the variables for frame size such as width and height of background frame

5. Perform the following processing starting form the second frame till the end of last frame in the video

A. Read the frame

B. Convert the frame to grey scale

C.Find the frame difference between current frame with previous frame

1. Diff frame $=$ frame $t-$ frame $t-1$

D.Classify the pixel whether it belongs to background or foreground

If the value of Diff_frame > Threshold value then

Pixel belongs to foreground

Store it in a new foreground vector array

Else

Set the corresponding foreground vector value to zero

End If

E.Now makes the current frame as previous frame and next frame as current frame 
6. Loop until end of the frame in the video.

\subsection{Feature Extraction}

The method of ruling image displacements which is easiest to understand is the feature-based approach. This finds features (for example, image edges, corners, and other structures well localized in two dimensions) and tracks these as they move from frame to frame. The act of feature extraction, if done well, will both reduce the amount of information to be processed (and so reduce the workload), and also go some way towards obtaining a higher level of understanding of the scene.

\subsubsection{Color Based Canny Edge Detection Method}

1. Read the input image

2. If the input image is a grayscale

a. Then simply apply the canny edge detection method

3. Else

4. Convert color image type from rgb to ycbcr

a. Then isolate the image into three color component

b. Apply the canny edge detection on first color component of the image

5. Then combine all the isolated components to a new image

6. End

The result shows the edge detection based on color.

\subsubsection{Object Detection Phase}

The object detection is performed by extracting the features of each object. Based on the dimension of every object it has its own specific feature. The feature extraction algorithm applied in this paper is optical flow which is used to detect and point out object in each frame sequence. In this method, the pixels are calculated based on the vector position and it is compared in frame sequences for the pixel position. In general the motion is correspond to vector position of pixels. Finding optic flow using edges has the advantage (over using two dimensional features) that edge detection theory is well superior, compared with that of two dimensional feature detection. It has the advantage over approaches which attempt to find flow everywhere in the object.

\subsubsection{Motion Estimation}

Optical flow is used to compute the motion of the pixels of an image sequence. It provides a dense (point to point) pixel correspondence. The problem is to determine where the pixels of an image at time $t$ are in the image at time $\mathrm{t}+1$. Large number of applications uses this method for detecting objects in motion.

\subsubsection{Optical Flow Algorithm}

Optical flow computation is based on two assumptions:

The experimental brightness of any object point is constant over time. Close to points in the image plane move in a similar manner (the velocity smoothness constraint). Suppose we have a continuous image; $f(x, y, t)$ refers to the gray-level of (x,y) at time t. Representing a dynamic image as a function of position and time permits it to be expressed.

- Assume each pixel moves but does not change intensity

- Pixel at location ( $x, y)$ in framel is pixel at $(x+\Delta x$, $\mathrm{y}+\Delta \mathrm{y})$ in frame2.

- Optic flow associates displacement vector with each pixel.

\subsubsection{Bayesian boosting for improving the performance of Optical flow technique (OFTBB)}

The Bayesian operator requires an example set as its input. To sample out prior knowledge of a different form it is possible to provide another model as an optional additional input. The predictions of this model are used to weight produce an initial weighting of the training set.

The output of the operator is a classification model which classifies the objects in the frame which is applicable for estimating conditional class probabilities or for plain crisp classification. It contains up to the specified number of inner base models. In the case of an optional initial model, this model will also be stored in the output model, in order to produce the same initial weighting during model application.

\subsection{Tracking}

Object tracking refers to the process of tracing the moving object in progression of frames. The task of tracking is performed by feature extraction of objects in a frame and discovering the objects in sequence of frames. By using the location values of object in each frame, we can determine the position and velocity of the moving object.

\subsubsection{Distance}

Using the centroid the distance traveled by the object is determined. The formula used for computing distance is Euclidean distance formula. The co-ordinate points are the pixel positions of moving object at first stage to the last stage.

Algorithm for calculating distance is explained as follow

- Find the centroid position of each object in the frame.

- Evaluate the distance between two centroid objects in the frame.

- For (current position=first value: last value) of $\mathrm{X}$ resolution

- For (current position=first value: last value) of $\mathrm{Y}$ resolution

- Calculate change in distance by

Distance $=\sqrt{\left(x_{2}-x_{1}\right)^{2}+\left(y_{2}-y_{1}\right)^{2}}$

Where

$$
\begin{aligned}
& \mathrm{X} 1=\begin{array}{c}
\text { preceding pixel position and } \mathrm{X} 2= \\
\text { current pixel position in width }
\end{array} \\
& \mathrm{Y} 1=\begin{array}{c}
\text { preceding pixel position and } \\
\text { current pixel position in height }
\end{array}
\end{aligned}
$$

- Store all the resultant distance values in an Array. 


\subsubsection{Velocity}

The velocity of moving object is calculated by the distance it traveled with respect to the time. Euclidean distance formula is used to calculate the distance between the sequences of frames. By using the values of distance with respect to frame rate, the velocity of the object is defined. The defined velocity is of 2-dimension

- Read the distance traveled by the object

- Velocity $=$ distance traveled/Video Frame rate

- $\quad$ Save the value in an array

- The velocity of moving object in the sequence frames is defined in pixels / second.

\section{EXPERIMENTAL RESULTS}

The experimental results are conducted with the help of MATLAB R2007a. Intel ${ }^{\circledR}$ Core $^{\text {TM}} 2$ DUO CPU T5870 and speed $2.00 \mathrm{GHZ}$ and its capacity is $2.99 \mathrm{~GB}$ of RAM. The performance of the object tracking is performed in four different phases and they are discussed below.

\subsection{Noise Removal Technique}

In this phase three different noises has been introduced on the video they are Gaussian noise, salt and pepper noise and Periodic Noise. Test was conducted on different filters among them the best suited filter are for Gaussian noise the wiener filter best suits, Salt and Pepper noise is effectively removed by Median filter and for the periodic noise 2D FIR filter performs better than other filters. The result obtained are shown in the figures.

Gaussian noise
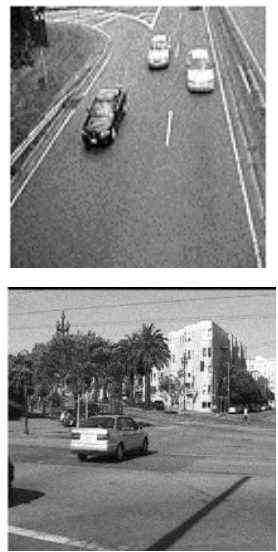

Salt And Pepper Noise
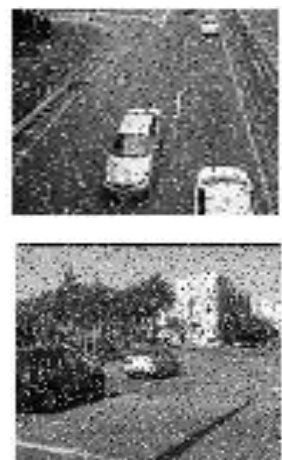

Periodic Noise
Wiener Filter
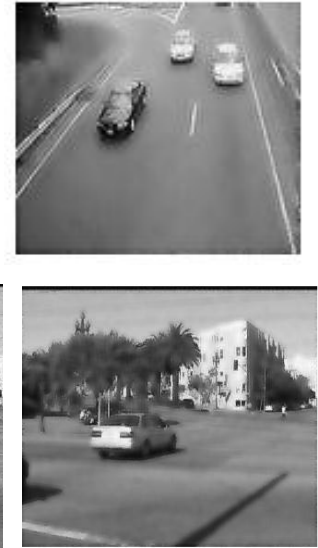

Median Filter
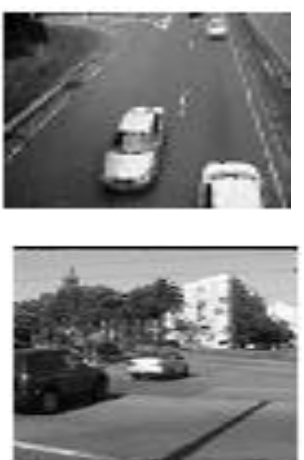

2D FIR Filter
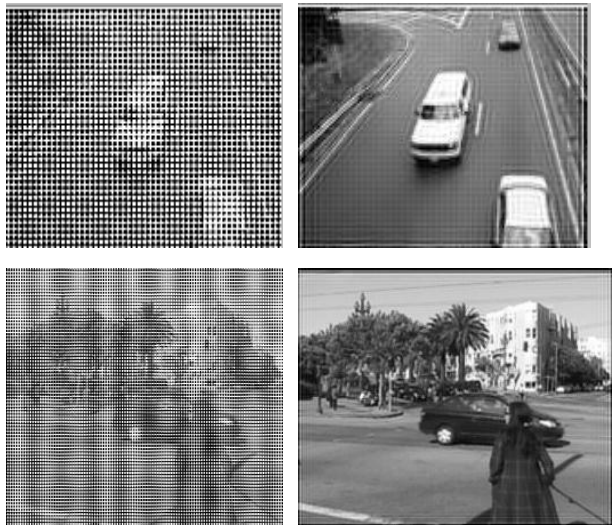

Figure: 2: Noise Removal Techniques for Various Noises

\subsection{Segmentation Technique}

The segmentation technique is used to group the similar objects by performing background subtraction using Frame difference. This technique best suited for moving objects segmentation. The result shows the input image, the previous frame and after applying the frame difference and subtracting the background objects the foreground is alone displayed the result is displayed in the figures.
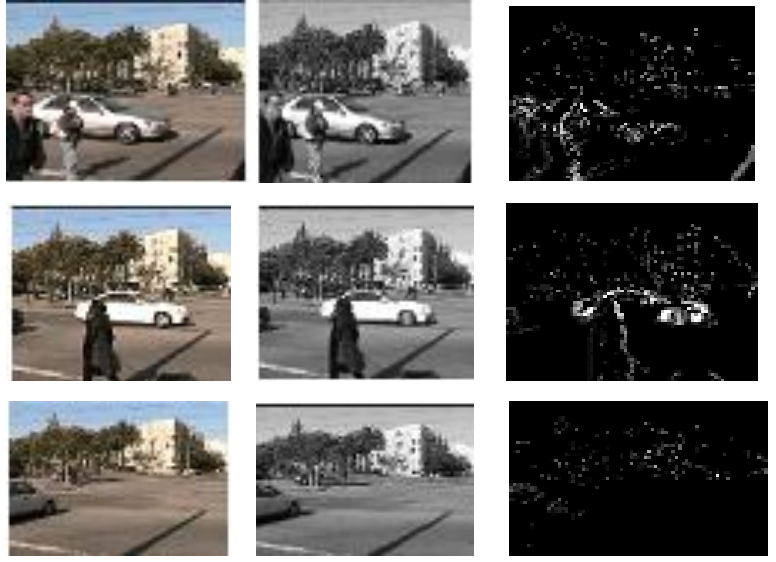

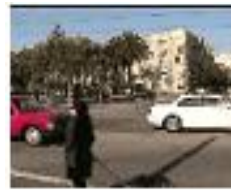

(a)

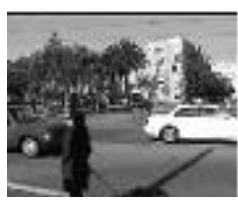

(b)

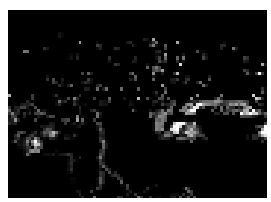

(c)
Figure: 3: Segmentation based on Frame difference in Traffic Video for various motion
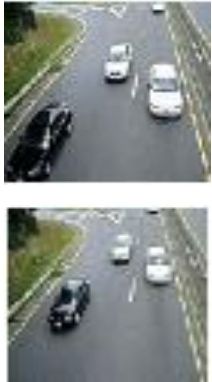

(a)
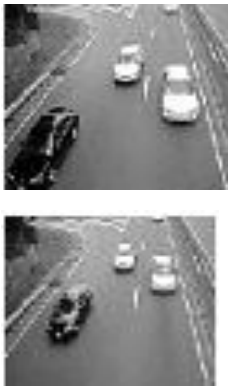

(b)
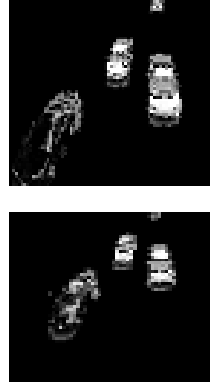

(c)
Figure: 4: Segmentation based on Frame difference 


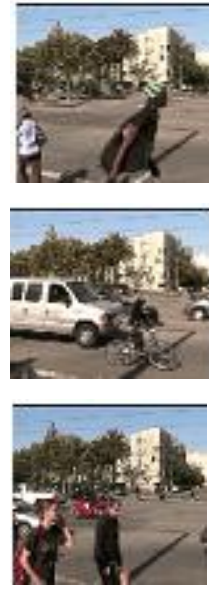

(a)

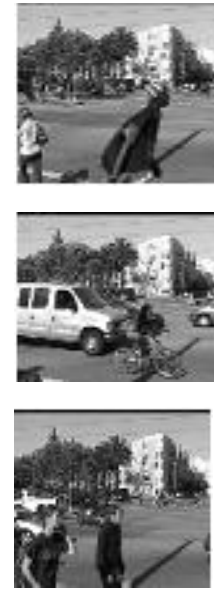

(b)
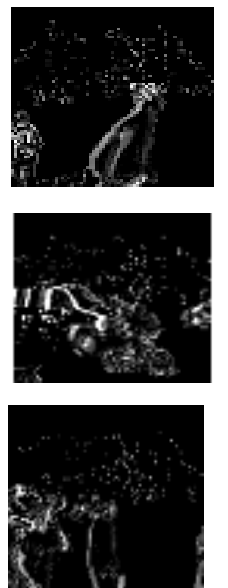

(c)
Figure: 5: Segmentation based on Frame difference

From the figure (a) shows the original video, (b) shows that the video is converted to grey scale and (c) shows the segmented output of the video in performing the frame difference of background subtraction.

\subsection{Feature extraction using Edge detection}

In order to detect moving objects in a video the feature extraction process plays a vital role for detecting moving objects in this paper edge detection is performed using color based canny edge detection method and the output of the segmented objected is displayed in the figure.
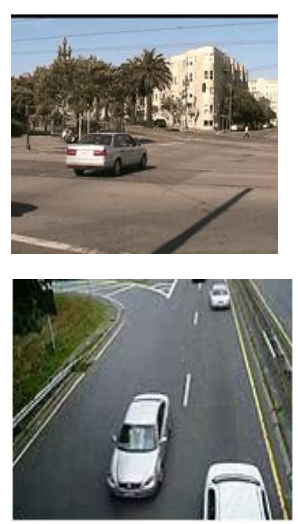

(a)
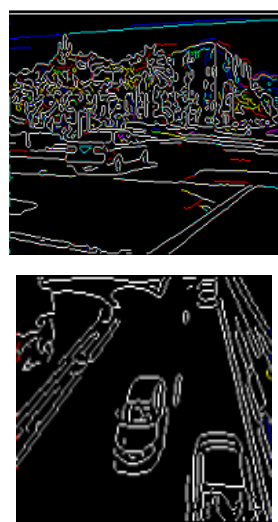

(b)
Figure: 6: Feature Extraction on Edge Detection Method

From the figure (a) shows the original video, (b) shows that the video of an moving object is detected using edge detection of Canny methods for various processing.

\subsection{Object Identification and Object Tracking}

Object tracking in video is performed by applying the optical flow method to set the motion vector of the moving objects then finding the threshold of each object and detecting and tracking the objects which exceeds the threshold value as moving objects. The experimental results are shown in the figure.

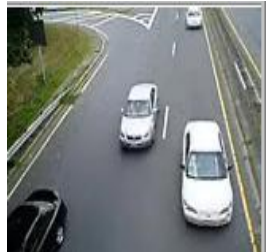

(a)

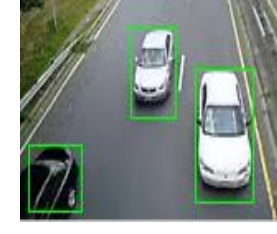

(c)

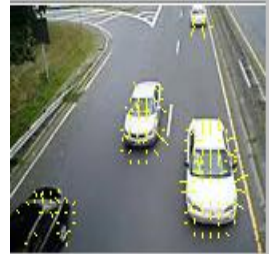

(b)

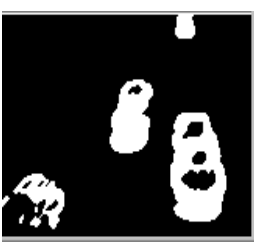

(d)
Figure: 7: Object Tracking and Detection using Optical Flow

From the figure (a) shows the original video, (b) shows optical flow method to set the motion vector, (c) shows the object and detecting and tracking the objects which exceeds the threshold value as moving objects and (d) Foreground of the moving object is detected.

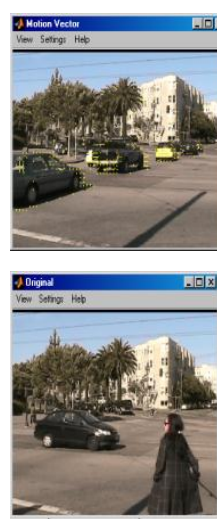

(a)
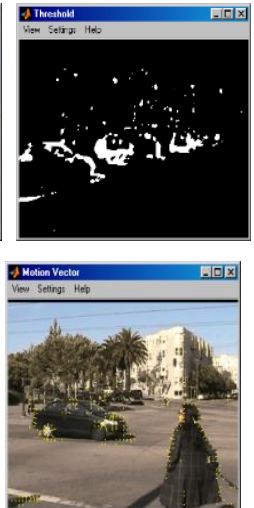

(b)

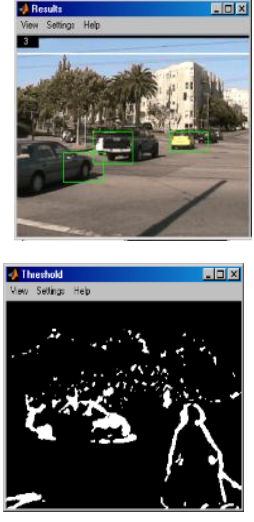

(c)

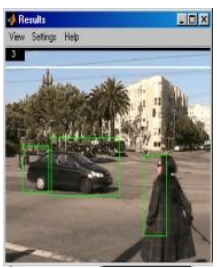

(d)

Figure: 8: Object Tracking and Detection using Optical Flow with Threshold Values

In the figure (a) shows the original video, (b) shows optical flow method to set the motion vector, (c) shows the object and detecting and tracking the objects which exceeds the threshold value as moving objects and (d) Foreground of the moving object is detected. 


\begin{tabular}{|c|c|c|c|c|}
\hline \multicolumn{5}{|c|}{ Moring Object } \\
\hline Algorithms & $\begin{array}{c}\text { Optical flor rector } \\
\text { computation }\end{array}$ & Segmentation & Tracking & $\begin{array}{c}\text { Execution } \\
\text { Time }\end{array}$ \\
\hline $\begin{array}{c}\text { Traditional. } \\
\text { optical Alow }\end{array}$ & 14.6583 & 35.6521 & 0.1456 & 50.456 \\
\hline OFTBB & 8.0256 & 19.5362 & 0.04256 & 27.60436 \\
\hline
\end{tabular}

From Table 1: It can be concluded that the optical flow with Bayesian boosting operator based Lucas approach gives better accuracy in vectors with reduced computation time in comparison with traditional optical flow based approach.

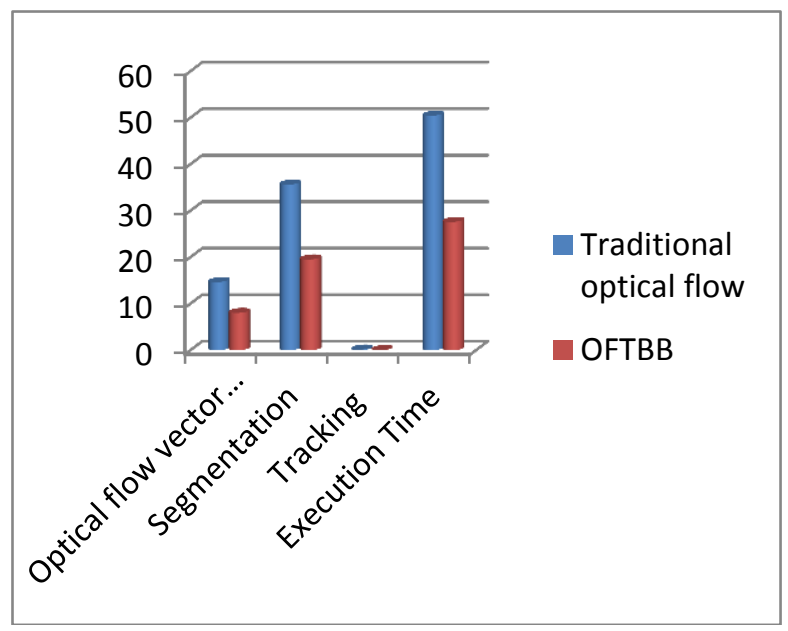

From Graph 1: It can be concluded that the optical flow with Bayesian boosting operator based Lucas approach gives better accuracy in vectors with reduced computation time in comparison with traditional optical flow based approach.

\section{CONCLUSION}

Moving object tracking is evaluated for various surveillance and vision analysis. The first task of this paper is to improve the quality of the video by applying noise removal on the input video for better result. Then the second phase is segmenting the object using background subtraction method with the help of finding frame difference method which gives a better understanding of grouping objects. In the third phase in order to determine the object in the frame feature extraction is performed using color based canny edge detection model. The moving objects in the frame are identified using the optical flow with Bayesian boosting algorithm method (OFTBB) which is our fourth phase. At last the moving object centroid is identified for calculating its distance and velocity. These algorithms can also be extended for the use of real-time applications and object classification.

The paper work can be extended using Artificial Intelligent and Fuzzy logic variance. These techniques can be able to handle the inconsistency and wagenence of segmenting object and tracking even when the quality of video is low.

\section{ACKNOWLEDGMENT}

I thank the Karpagam University for the motivation and Encouragement for giving me the opportunity to do this research work as successful one.

\section{REFERENCES}

[1] Peter Mountney, Danail Stoyanov and Guang-Zhong Yang (2010). "Three-Dimensional Tissue Deformation Recovery and Tracking: Introducing techniques based on laparoscopic or endoscopic images." IEEE Signal Processing Magazine. 2010 July. Volume: 27". IEEE Signal Processing Magazine 27 (4): 14-24. DOI:10.1109/MSP.2010.936728.

[2] Lyudmila Mihaylova, Paul Brasnett, Nishan Canagarajan and David Bull (2007). Object Tracking by Particle Filtering Techniques in Video Sequences; In: Advances and Challenges in Multisensor Data and Information. NATO Security Through Science Series, 8. Netherlands: IOS Press. pp. 260-268. ISBN 978-158603-727-7.

[3] Kato, Hirokazu, and Mark Billinghurst (1999). "Marker Tracking and HMD Calibration for a Video-based Augmented Reality Conferencing System". IWAR '99 Proceedings of the 2nd IEEE and ACM International Workshop on Augmented Reality (IEEE Computer Society, Washington, DC, USA).

[4] A.Gyaourova, C.Kamath,S.and C.Cheung-Block Matching object tracking-LLNL Technical report, October 2003.

[5] Y.Rosenberg and M.Weman-Real Time Object Tracking from a Moving Video Camera: A software approach on PC-Applications of Computer Vision „1998.WACV '98.Proceedings.

[6] A.Turolla,L.Marchesotti and C.S.RegazzoniMulticamera Object tracking in video surveillance applications.

[7] Y.Wang,J.Doherty and R.Van Dyck-Moving object tracking in video-Proc. Conference on Information Sciences and Systems,Princeton,NJ,March2000.

[8] Ci gdem Ero glu Erdem and Bulent San-Video Object Tracking With Feedback of Performance Measures-IEEE Transactions on circuits and systems for video technology,vol.13,no.4,April 2003.

[9] Alok K.Watve and Shamik Sural - A Seminar on Object tracking in Video Scenes.

[10] P.Subashini,M.Krishnaveni,Vijay Singh-Implementation of Object Tracking System Using Region Filtering Algorithm based on Simulink Blocksets, International Journal of Engineering Science and Technology(IJEST),Vol.3 No.8 August 2011. PP-67446750.ISSN:0975-5462.

[11] Ashwani Aggarwal,Susmit Biswas,sandeep Singh,Shamik Sural and A.K.Majundar,-Object tracking using background subtraction and motion estimation in MPEG videos,Springer-Verlag Berlin Heidelberg,ACCV 2006,LNCS 3852,pp:121-130.

[12] Zhuohua Duan,Zixing Cain ad Jinxia Yu,'Occlusion detection and recovery in video object tracking based on adaptive particle filters,"IEEE trans.China,PP.466469[Chinese Control and Decision Conference CCDC2009].

[13] XI Tao,Zhang shengxiu and Yan shiyuan,"A robust visual tracking approach with adaptive particle filtering,"IEEE Second Intl.Conf. on Communication Software and Networks 2010,pp:549-553. 
[14] Xiaoqin Zhang,Weiming Hu,Zixiang zhao, Yan-guo Wang,Xi Li and Qingdi Wei,"SVD based kalman particle filter for robust visual tracking," IEEE 2008,978-1-42442175-6.

[15] Li Ying-hong,Pang Yi-gui,Li Zheng-xi and Liu Yali,"An intelligent tracking technology based on kalman and mean shift algorithm," IEEE Second Intl.Conf. on Computer Modeling and Simulation 2010.

[16] Madhur Mehta,Chandni Goyal,M.C.Srivastava and R.C.Jain,"Real time object detection and tracking:Histogram matching and kalman filter approach," IEEE 2010,978-1-4244-5586-7.

[17] A.Purushothaman,K.R.Shankar kumar,R.Rangarajan,A.Kandasawamy-“Compressed Novel Way of Tracking Moving Objects in Image and Video Scenes,"European Journal of Scientific Research ,Vol.64 No.3(2011),pp.353-360.ISSN 1450-216X.

[18] G.Suresh, P.Epsiba,

Dr.M.Rajaram, Dr.S.N.Sivanandam," Image And Video Coding With A New Wash Tree Algorithm For Multimedia Services", Journal of Theoretical and Applied Information Technology, 2005 - 2009, PP: 53-59.

[19] T. Senthil Kumar, S. N. Sivanandam," A Modified Approach for Detecting Car in video using Feature
Extraction Techniques", European Journal of Scientific Research, ISSN 1450-216X Vol.77 No.1 (2012), pp.134144.

[20] Daniel Marcus jang,Matthew Turk,"CarRec: A Real Time Car Recognition System", WACV '11 Proceedings of the 2011 IEEE Workshop on Applications of Computer Vision.

[21] Luo Juan, Oubong Gwun "A Comparison of SIFT, PCASIFT and SURF", International Journal of Image Processing (IJIP), september 10,2008.

[22] T.Senthil kumar, Dr.S.N.Sivanandam, Adheen Ajay, and P. Krishnakuma," An improved approach for Character Recognition in Vehicle Number plate using Eigenfeature Regularisation and Extraction Method", International Journal of Research and Reviews in Electrical and Computer Engineering (IJRRECE) Vol. 2, No. 2, June 2012, ISSN: 2046-5149.

[23] X. Q. Ding, "Machine printed Chinese character recognition," in Handbook of Character Recognition and Document Image Analysis, H. Bunke and P.S.P. Wang, Beijing: World Scientific Publishing Company, 1997, pp. 305-329

[24] Spector, A. Z. 1989. Achieving application requirements. In Distributed Systems, S. Mullender. 